\title{
A NOTE ON LOCALLY CONSTANT INCOME ELASTICITIES
}

\author{
Hal R. VARIAN * \\ University of Michigan, Ann Arbor, MI 48104, USA
}

Received April 1978

\begin{abstract}
A simple proof of Willig's theorem that all non-unitary income elasticities that are constant must be equal to one another is given. It is also shown that multiplicatively separable demand implies locally constant income elasticity.
\end{abstract}

Robert Willig has recently proved the following remarkable theorem: 'all nonunitary income elasticities of an individual's demands that are constant must be equal to one another' [Willig (1976a)]. His proof is logically straightforward, but rather complex in detail. It involves reconstructing the indirect utility function by integrating the compensated demand functions.

In this note I provide a simple proof of Willig's theorem based on Slutsky's equations. Before proceeding to that argument, it is worthwhile to point out the following simplc but interesting fact:

Proposition. Suppose that the demand function for some good $i$ can be written in the form $X_{i}(p, y)=x_{i}(p) g_{i}(y)$ for $(p, y)$ lying in some open set in $R^{k+1}$. Then in fact $g_{i}(y)=y^{\eta_{i}}$; i.e., the demand function must have a locally constant income elasticity.

Proof. Since demand functions are homogeneous of degree zero in $(p, y)$ we can apply Euler's law to get

$g_{i}(y) \sum_{j=1}^{k} \frac{\partial x_{i}}{\partial p_{j}} p_{j}+x_{i} \frac{\mathrm{d} g_{i}}{\mathrm{~d} y} y=0$,

or

$\left(\sum_{j=1}^{k} \frac{\partial x_{i}}{\partial p_{j}} p_{j}\right) / x_{i}=-\frac{\mathrm{d} g_{i}}{\mathrm{~d} y} \frac{y}{g_{i}(y)}$.

* I wish to thank Theodore Bergstrom and Robert Willig for some helpful conversations concerning this note. 
The right-hand side is a function of $y$ alone, and the left-hand side is a function of $p$ alone. Hence both sides of the identity must be constant. But the right-hand side of this identity is simply the income elasticity of demand. ${ }^{1}$

The empirical implications of this proposition are rather striking. The multiplicative separability of demand property is an empirically testable hypothesis - one simply tests for the absence of a price-income interaction. If one can reject the null hypothesis of significant interaction, one can postulate an exact functional form for how income enters the demand function. If the data indicate that the constant income elasticity hypothesis is a reasonable functional form for at least two demanded goods, then one can invoke the strong integrability restrictions derived by Willig.

As mentioned earlier, Willig's original argument was fairly complex. It stands to reason that a simpler argument should be available just by examining the restrictions imposed by the Slutsky equations when income elasticities are constant. For the basic result of integrability theory is that the Slutsky restrictions are a complete list of the restrictions imposed by utility maximizing behavior. Hence any cross equation restrictions imposed by choice of functional form should be extractable from the Slutsky restrictions alone. This insight, plus a few pads of yellow paper, led to the following argument:

Theorem. Suppose that the demand functions for two goods can be written as

$$
\begin{aligned}
& X_{1}(p, y)=x_{1}\left(p, y_{0}\right)\left[y / y_{0}\right]^{\eta_{1}}, \\
& X_{2}(p, y)=x_{2}\left(p, y_{0}\right)\left[y / y_{0}\right]^{\eta_{2}},
\end{aligned}
$$

for some open set $(p, y)$ in $\boldsymbol{R}^{k+1}$ that contains $y_{0}$. Then either:

$$
\begin{array}{ll}
\text { (1) } \eta_{1}=\eta_{2}, & \partial x_{1} / \partial p_{2}=\partial x_{2} / \partial p_{1} ; \\
\text { (2) } \eta_{1}=1, & \partial x_{1} / \partial p_{2}=0 ; \quad \text { or } \\
\text { (3) } \eta_{2}=1, & \partial x_{2} / \partial p_{1}=0 .
\end{array}
$$

Proof. For notational convenience, let $m=y / y_{0}$. The Slutsky symmetry condition can be written as

$\frac{\partial x_{1}}{\partial p_{2}} m^{\eta_{1}}+\frac{\eta_{1} x_{1} m^{\eta_{1}-1} x_{2} m^{\eta_{2}}}{y_{0}}=\frac{\partial x_{2}}{\partial p_{1}} m^{\eta_{2}}+\frac{\eta_{2} x_{2} m^{\eta_{2}-1} x_{1} m^{\eta_{1}}}{y_{0}}$,

1 This fact was pointed out to me by my colleague Theodore Bergstrom. However, the argument is a direct adaptation of Willig's (1976a) argument (Theorem 3). 
or

$\frac{\partial x_{1}}{\partial p_{2}} m^{\eta_{1}}=\frac{\partial x_{2}}{\partial p_{1}} m^{\eta_{2}}+\frac{x_{1} x_{2}}{y_{0}} m^{\eta_{1}+\eta_{2}-1}\left(\eta_{2}-\eta_{1}\right)$.

Multiply by $m^{-\eta_{1}}$,

$\frac{\partial x_{1}}{\partial p_{2}}=\frac{\partial x_{2}}{\partial p_{1}} m^{\eta_{2}-\eta_{1}}+\frac{x_{1} x_{2}}{y_{0}} m^{\eta_{2}-1}\left(\eta_{2}-\eta_{1}\right)$.

Differentiate twice with respect to $m$,

$$
\begin{aligned}
0= & \frac{\partial x_{2}}{\partial p_{1}}\left(\eta_{2}-\eta_{1}\right) m^{\eta_{2}-\eta_{1}-1}+\frac{x_{1} x_{2}}{y_{0}}\left(\eta_{2}-1\right) m^{\eta_{2}-2}\left(\eta_{2}-\eta_{1}\right) \\
0= & \frac{\partial x_{2}}{\partial p_{1}}\left(\eta_{2}-\eta_{1}\right)\left(\eta_{2}-\eta_{1}-1\right) m^{\eta_{2}-\eta_{1}-2} \\
& +\frac{x_{1} x_{2}}{y_{0}}\left(\eta_{2}-1\right)\left(\eta_{2}-2\right) y^{\eta_{2}-3}\left(\eta_{2}-\eta_{1}\right)
\end{aligned}
$$

Evaluate expression (2) at $y=y_{0}, m=1$,

$0=\frac{\partial x_{2}}{\partial p_{1}}\left(\eta_{2}-\eta_{1}\right)+\frac{x_{1} x_{2}}{y_{0}}\left(\eta_{2}-1\right)\left(\eta_{2}-\eta_{1}\right)$,

$0=\left(\eta_{2}-\eta_{1}\right)\left[\frac{\partial x_{2}}{\partial p_{1}}+\frac{x_{1} x_{2}}{y_{0}}\left(\eta_{2}-1\right)\right]$.

If $\eta_{2}=\eta_{1}$ we are done. [Expression (1) shows that $\partial x_{2} / \partial p_{1}=\partial x_{1} / \partial p_{2}$.] Otherwise we have

$\frac{\partial x_{2}}{\partial p_{1}}=-\left(\eta_{2}-1\right) \frac{x_{1} x_{2}}{y_{0}}$.

Evaluate expression (3) at $y=y_{0}, m=1$, and use expression (4) to get

$0=-\left(\eta_{2}-1\right) \frac{x_{1} x_{2}}{y_{0}}\left(\eta_{2}-\eta_{1}\right)\left(\eta_{2}-\eta_{1}-1\right)+\frac{x_{1} x_{2}}{y_{0}}\left(\eta_{2}-1\right)\left(\eta_{2}-2\right)\left(\eta_{2}-\eta_{1}\right)$.

Since we are considering the case where $\eta_{2} \neq \eta_{1}$ we can factor out $\left(\eta_{2}-\eta_{1}\right)$. Since $x_{1} x_{2}>0$ we can factor out $x_{1} x_{2} / y_{0}$. If we assume $\eta_{2} \neq 1$, then we can factor it out and be left with

$-\left(\eta_{2}-\eta_{1}-1\right)+\left(\eta_{2}-2\right)=0$,

or

$\eta_{1}=1$. 
By symmetry, if $\eta_{1} \neq 1$ and $\eta_{1} \neq \eta_{2}$, then $\eta_{2}=1$. Using expression (4) and the symmetric expression for $\partial x_{1} / \partial p_{2}$ we have the result. ${ }^{2}$

Given the strong integrability restrictions on demand behavior, it is straightforward to derive the form of the indirect utility function. For notational simplicity we suppose that goods 1 and 2 have constant income elasticities over some region of the price space. We let $\left(\bar{p}_{1}, \bar{p}_{2}\right)$ be upper bounds on these prices and let $q$ be the fixed vector of other prices. ${ }^{3}$ Now define the function

$A\left(p_{1}, p_{2}\right)=\int_{p_{1}}^{\bar{p}_{1}} x_{1}\left(t_{1}, p_{2}, q, y_{0}\right) \mathrm{d} t_{1}+\int_{p_{2}}^{\bar{p}_{2}} x_{2}\left(\bar{p}_{1}, t_{2}, q, y_{0}\right) \mathrm{d} t_{2}$.

This is just the multidimensional consumer's surplus between $\left(p_{1}, p_{2}\right)$ and $\left(\bar{p}_{1}, \bar{p}_{2}\right)$. We have defined the surplus along a particular path, but the fact that $\partial x_{1} / \partial p_{2}=$ $\partial x_{2} / \partial p_{1}$ implies that in fact this line integral is path independent.

Now it is easy to see that the indirect utility function must have the form

$$
\begin{aligned}
v\left(p_{1}, p_{2}, q, y\right) & =G\left[A\left(p_{1}, p_{2}\right)+\frac{y^{1-\eta}}{1-\eta}, q\right], & & \eta \neq 1, \\
& =G\left[A\left(p_{1}, p_{2}\right)+\ln y, q\right], & & \eta=1,
\end{aligned}
$$

where $q$ is the vector of $k-2$ other prices and $G$ is some monotonic transform that may depend on $q$. The proof is simply to note that the application of Roy's identity to (5) or (6) yields the original constant income elasticity demands

$X_{1}(p, y)=\frac{-\partial v / \partial p_{1}}{\partial v / \partial y}=\frac{\partial A / \partial p_{1}}{y^{-\eta}}=x_{1}\left(p, y_{0}\right) y^{\eta}$

(Willig derives this result in the aforementioned paper by a different and more rigorous argument.)

Given that we know the form of the indirect utility function, it is casy to make direct welfare comparisons of different price income regimes. By definition of the indirect utility function, $\left(p^{0}, y^{0}\right)$ is preferred to $\left(p^{\prime}, y^{\prime}\right)$ if and only if $v\left(p^{0}, y^{0}\right)>v\left(p^{\prime}, y^{\prime}\right)$.

If the only prices that have changed are the prices of two goods that have constant income elasticities, we can apply the above results to see that $v\left(p^{0}, y^{0}\right)>v\left(p^{\prime}, y^{\prime}\right)$

2 Note that this argument only requires that $\eta_{1}$ and $\eta_{2}$ be independent of $p_{1}$ and $p_{2}$; that is, the income elasticities may be a function of the $k-2$ other prices.

3 Note that $\eta_{1}$ and $\eta_{2}$ may depend on $q$. Also, in what follows we will ignore the possibilities described in parts (2) and (3) of the above theorem. 
if and only if

$$
\begin{array}{cc}
\int_{p_{1}}^{p_{1}^{\prime}} x_{1}\left(t_{1}, p_{2}^{0}, y^{0}\right) \mathrm{d} t_{1}+\int_{p_{2}}^{p_{2}^{\prime}} x_{2}\left(p_{1}^{\prime}, t_{2}, y^{0}\right) \mathrm{d} t_{2}>\frac{\left(y^{\prime}\right)^{1-\eta}-\left(y^{0}\right)^{1-\eta}}{1-\eta}, & \eta \neq 1 \\
& >\ln y^{\prime}-\ln y^{0}, \quad \eta=1 .
\end{array}
$$

That is, utility has increased if and only if the change in consumer's surplus exceeds the appropriate function involving the change in income. If the income elasticity is zero this reduces to the standard consumer's surplus comparison. If the income elasticity is non-zero but constant, the above formulas provide exact comparisons. If the income elasticities are non-constant, Willig (1976b) has shown how one can bound the consumer's surplus error by a function of observable upper and lower bound on the income elasticities.

\section{References}

Willig, R., 1976a, Integrability implications for locally constant demand elasticities, Journal of Economic Theory 12, 391-401.

Willig, R., 1976b, Consumer's surplus without apology, American Economic Review 66, 589-597. 